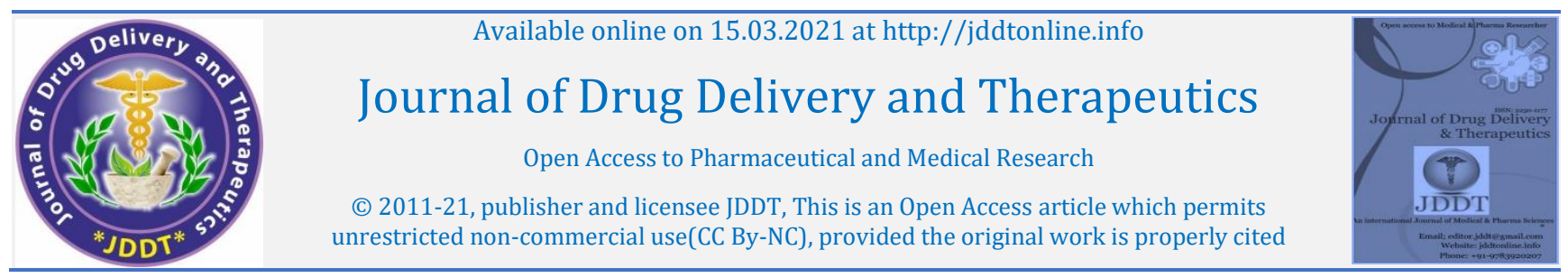

(C) 2011-21, publisher and licensee JDDT, This is an Open Access article which permits Open Access Full Text Article

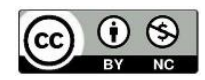

Review Article

\title{
Nano Sponges: A Novel Approach for Targeted Drug Delivery Systems
}

\author{
K. Muni Raja Lakshmi*, CH. Suma Shree, N. Lakshmi Priya \\ Department Pharmaceutics, Sri Venkateswara University, Tirupati, Andhra Pradesh, India
}

\section{Article Info:

$\begin{array}{ll} & \text { Article History: } \\ & \text { Received 12 Jan 2021; } \\ & \text { Accepted 08 March 2021; } \\ & \text { Available online 15 March } 2021\end{array}$

Cite this article as:

Muni Raja Lakshmi K, Suma Shree C, Lakshmi Priya N, Nano Sponges: A Novel Approach for Targeted Drug Delivery Systems, Journal of Drug Delivery and Therapeutics. 2021; 11(2):247-252

DOI: http://dx.doi.org/10.22270/jddt.v11i2.4612

\section{*Address for Correspondence:}

Dr. K. Muni Raja Lakshmi, SVU College of Pharmaceutical Sciences, SV University, Tirupati 517501, Andhra Pradesh, India.

\section{Abstract}

Targeted drug delivery system is a unique type of medication conveyance framework where the pharmacologically dynamic specialists mainly focused on its site activity and not to the non-focused on organs, tissues or cells. Nano sponges are such sort of compelling pharmaceutical transporters, which take care of issues like harmfulness and helpless bioavailability such as they can stack both hydrophilic and hydrophobic medicines. Various classifications of medications can stack into Nano sponge for focused medication conveyance. This targeted drug delivery system is one of the most encouraging methodologies in the existence of science. Nano sponges are little in size with a three-dimensional organization and nanometric depression. Nano sponges are exceptionally permeable and have the extraordinary capacity to ensure dynamic particles and offer programmable delivery. This Review article explains the overall presentation of nano sponges a grouping of nano sponge trademark highlights of nano sponges their points of interest inconveniences, and synthetic compounds utilized in their planning, arrangement of technical factors influencing on the preparation component activities and assessment boundaries with some particular applications.

Keywords: Nano sponges; Targeted drug delivery; Solubility Enhancement; controlled drug delivery.

\section{Introduction:}

Nanotechnology expresses the deceive of circumstances on an atomic, molecular, and super molecular scale requiring the plan, manufacturing, evaluation and implementation of variant nano-scale materials in variant probable areas gives novel technological advances mainly in the field of medicine. Nanotechnology has provided probable impact in different fields like medicine including immunology, cardiology, endocrinology, ophthalmology, oncology, pulmonology etc... In incorporation it's highly used in specialized areas like brain targeting, tumour targeting, and gene delivery. Nanotechnology also dispenses important systems, devices and materials for better pharmaceutical applications 1 .

Nano sponge is a current class of material and is made up of small particles with a limited space These limited spaces are filled with variety of substances. These small particles are having a capacity to carry both hydrophilic and lipophilic molecules and can improve the stability of poorly watersoluble molecules. ${ }^{2}$

The pharmaceutical and health care industry has been providing and utilising nano-scale data for solving many physical, chemical and biological problems analogues with the therapy of ailment. Since the 1950's, nanotechnology has dominated technology.$^{3}$

1.1. Encapsulating Nanoparticles: This type is represented by nano sponges and nano capsules. Nano sponges such as alginate Nano sponges, which are sponge like nanoparticles accommodate many hollows that bring the drug molecules. Nano capsules such as poly (isobutyl cyanoacrylate) (IBCA) are also encapsulating nanoparticles. They can include drug molecules in their watery core.

1.2. Complexion Nanoparticles: This class includes compound nanoparticles, which allure the molecules by electric charges.

1.3. Conjugating Nanoparticles: These combinations of nanoparticles link to drugs through covalent bonds . 4

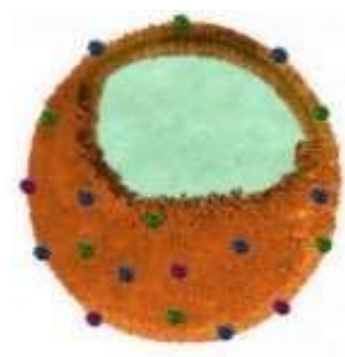

Figure1: Structure of a nanosponge demonstrating a depression for drug stacking

\section{Advantages:}

- $\quad$ Efficient capture of fixings and diminished results. 
- Improved security, expanded polish and upgraded detailing adaptability.

- These details are steady up to a temperature of $130^{\circ} \mathrm{C}$.

- $\quad$ These definitions are viable with most vehicles and fixings.

- $\quad$ These are self-disinfecting as their normal pore size is $0.25 \mu \mathrm{m}$ which makes the microbes unfit to enter.

- $\quad$ These are free streaming and can be practical.

- These definitions alter the arrival of the medication.

- They increment the solvency of ineffectively dissolvable medication.

- It can be utilized to veil flavours and to change fluid substance over to sol. 5

\section{Disadvantages:}

- Nano sponges have the limit of typifying little particles, not reasonable for bigger atoms.

- Dose unloading may happen at time

- Nano sponges have capacity to incorporate just little atoms.

- Nano sponges could be either Para translucent or in glasslike structure.

- The stacking limit of nano sponges relies predominantly upon level of crystallization.$^{5}$

\section{Materials and Methods of Nano sponges:}

4.1. Polymer: The determination of polymer can impact the arrangement alongside the exhibition of Nano sponges. The cavity size should be appropriate to consolidate the specific medication particle. The polymer determination depends on the necessary delivery and medication to be encased. They chose polymer ought to have the property to append with explicit legends.

4.2. Cross connecting specialist: The crosslinking specialist determination can be done relying on the structure of polymer and the medication which is to be defined. The various models incorporate Diphenyl carbonate, Dichloromethane, Daryl carbonates, Diisocyanatos.$^{6}$

4.3. Drug substance: Molecular load somewhere in the range of 100 and 400 Daltons. Drug particle comprises of under-five consolidated rings solubility in water is under 10 $\mathrm{mg} / \mathrm{ml}$.

\section{Method of preparation:}

5.1. Dissolvable technique: Mix the polymer with an appropriate dissolvable, specifically polar aprotic dissolvable, for example, Dimethyl formamide, Dimethyl sulfoxide. At that point add this combination to overabundance amount of the cross linker, ideally in cross linker polymer molar proportion of 4 to 16 . Complete the response at temperature going from $100 \mathrm{C}$ to the reflux temperature of the dissolvable, for time going from 1 to 48 hrs.

Favoured cross linkers are carbonyl mixes (Dimethyl carbonate and Carbonylic imidazole). After finish of the response, permit the answer for cool at room temperature, at that point add the item to enormous abundance of stilled water and recuperate the item by filtration under vacuum and hence refine by delayed Soxhlet.

5.2. Nano sponges produced using hyper crossconnected $\boldsymbol{\beta}$-cyclodextrins: The $\beta$-cyclodextrin Nano sponges to hold both hydrophilic drugs, and lipophilic drugs, Lipophilic drugs has a larger drug holding tendency than hydrophilic one because of their bigger number of water insoluble areas obtainable for drug complexation. Moreover, the drug holding tendency seemed to be relying on the artificial route selected: The Nano sponges was obtained by mixing like sonication, was used, to get bigger substance size obtained. ${ }^{7}$

5.3. Ultrasound-Assisted Synthesis: In this strategy, polymers respond with cross-linkers without dissolvable and under sonication. Here, blend the polymer and cross-linker in a cup. Spot the flagon in an ultrasound shower loaded up with water and warmth it to $90 \mathrm{o} \mathrm{C}$ and sonicate for 5 hours. Permit it to cool and wash with water to eliminate the nonreaction polymer. Clean by delayed Soxhlet extraction with ethanol. Dry the item under vacuum and store.$^{8}$

5.4. Emulsion Solvent Diffusion Method: Nano sponges can be set up by utilizing ethyl cellulose (EC) and polyvinyl liquor (PVA). Ethyl cellulose is broken up in dichloromethane. Add this combination into watery arrangement of polyvinyl liquor. Mix the combination at $1000 \mathrm{rpm}$ for 2 hours in an attractive stirrer. At that point channel the item and dry it in a broiler at 40 o C for 24 hours 9

Table: 1 Materials used in the preparation of nanosponges

\begin{tabular}{|l|l|l|}
\hline Polymer & Copolymer & Cross linker \\
\hline Hyper cross linkedpolystyrene & Ethyl cellulose, & Dicarbonate, \\
\hline $\begin{array}{l}\text { Cyclodextrins and its derivatives like Methyl ßcyclodextrine, } \\
\text { 2hydropropyl } \beta \text { cyclodextrine }\end{array}$ & poly vinyl alcohol [PVA] & Di- isocyanate \\
\hline
\end{tabular}




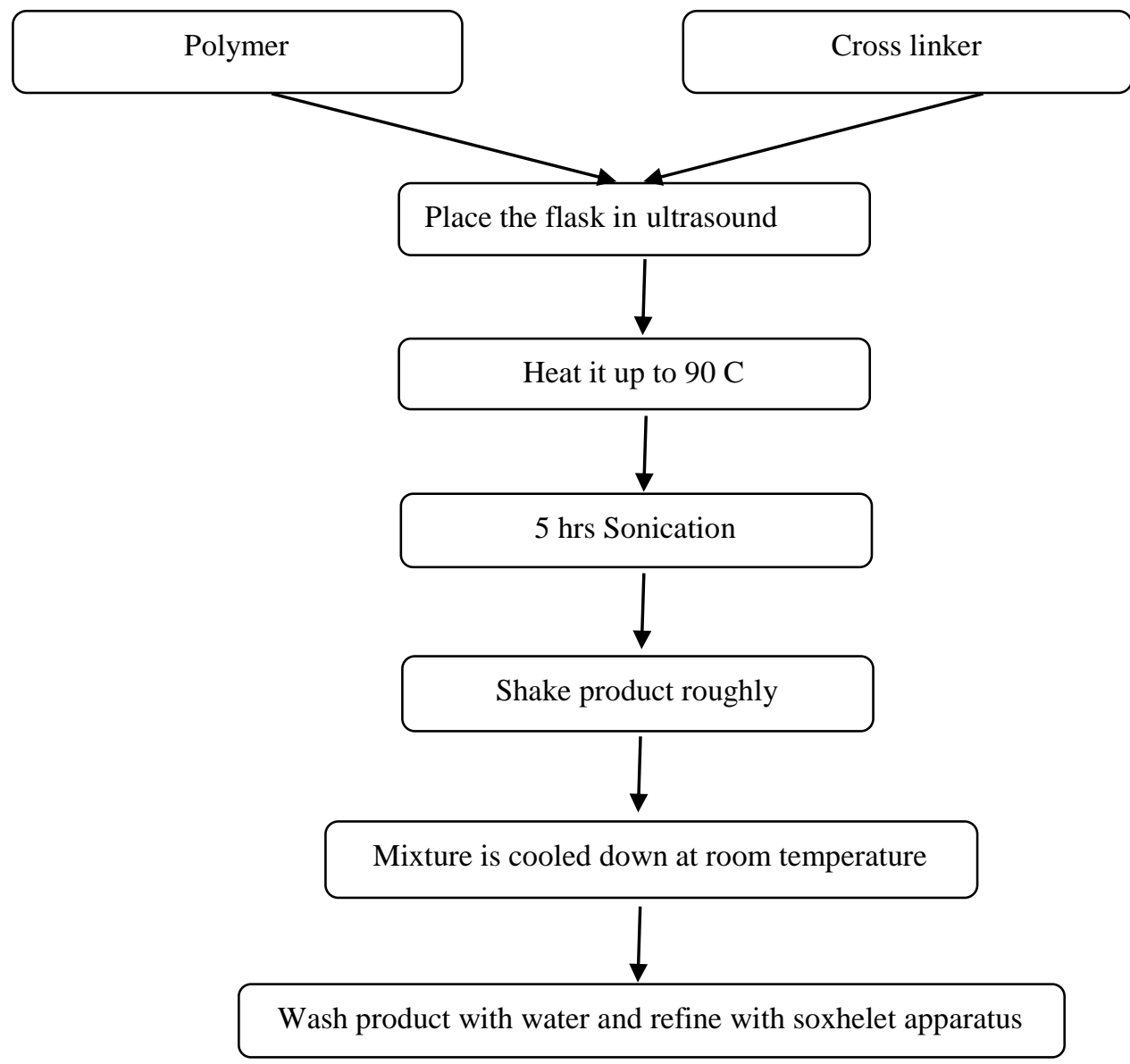

Flow Chart 1: For the preparation of nanosponges using ultra assisted method

Organic internal phase containing drug and polymer in solvent is added

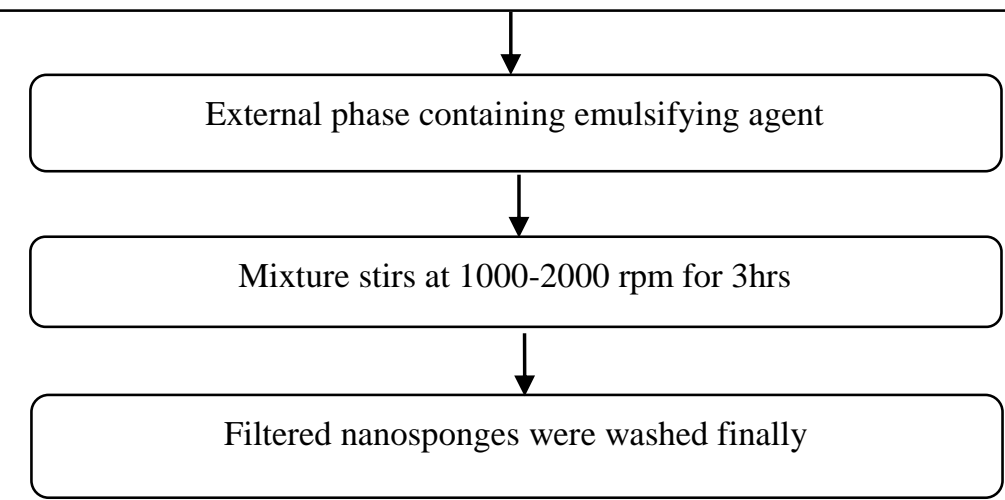

Flow diagram 2: The preparation of nanosponges using emulsion solvent diffusion method

\section{Loading of Nano sponges}

Conveying of medication stacked nano-transporters to the objective site can be accomplished by 3 unique instruments: uninvolved, dynamic and truly focusing on. Aloof focusing on prompts an expanded penetrability and maintenance (EPR) impact, which permits tumour cells to ingest ideally NC-sized bodies. Albeit most tumours fluctuate in pore size contingent upon tumour type, they are accounted for to have a lot bigger pores (about 380_780 $\mathrm{nm}$ ) than solid organs Therefore, the size of the readied nano structure is significant for the amassing of the tumoral locale by the impact of EPR in the vessel dividers and penetrability to the tumour vascular framework.

The dynamic focusing on approach is a strategy for directing surface changes to nanocarriers utilizing certain ligands like antibodies, sugars, folic corrosive, peptides, and acquainting and restricting ligand nanostructures with a specific tumour cell receptor. Physical focusing on is a technique for guiding NCs to the objective site and controlling the delivery interaction utilizing outside sources or territories, for instance photothermal and attractive field. ${ }^{10}$ 


\section{Mechanism of medication discharge from Nano sponges:}

Since the nano sponges have an open structure (in the encompassing of nano sponges they don't have any consistent film), the dynamic substance is added to the vehicle in an epitomized structure. The exemplified dynamic substance can move unreservedly from the particles into the vehicle until the vehicle gets immersed and the harmony is gotten. When the item is applied on to the skin, the vehicle in the balance.

In this way, the progression of dynamic substances from nano sponge particles into vehicles starts to epidermis until the vehicle is either ingested or dried. Even after the maintenance of the nano wipe particles on the outside of skin for example the layer corneum, the arrival of dynamic substance keeps on cleaning for a significant stretch of time.

\section{Factors affecting in the definition of nano sponges:}

8.1 Nature of polymer: The polymer utilized in the readiness of nano sponges can impact its development and can likewise influence the pre-plan. The size of the depression of a nano sponge should be large enough to ensnare a medication.$^{11}$

8.2 Drug: To be mind boggling with nano sponges, the medication atoms ought to have some particular attributes as referenced underneath. Changes in the temperature can influence the complexation of medication or nano sponges. Expanding the temperature for the most part diminishes the perplexing which might be because of the decrease of communication powers, for example, hydrophobic powers and Vander Waal powers of medication/nano sponges with an expansion in the temperature.

8.3 Complexation Temperature: The durable of a complex is dependent on temperature changes. The durable kinetics and heat increases are reversely correlated. At rise in temperature, the immensity of actual strongness undergoes down due to less in medication/nano sponge forces. Although maintain temperature must be necessary for preparation of nanosponges. ${ }^{12}$

8.4 Degree of Substitution: The number, sort and posture of the replacement on the polymer material influence the compound capacity of nano sponges. The sort of the replacement is necessary because CD derivatives accessible in different forms of functional categories .12

\section{Types of Nano sponges:}

CD based NS are further classified into:

\subsection{CD-based carbonate nano sponges:}

\section{Types of Nano sponges:}

CD based NS are further classified into:

\subsection{CD-based carbonate nano sponges:}

These were blended Cyclodextrin based carbonate nano sponges beginning from local $\beta$-cyclodextrin and dynamic carbonyl mixtures for example Carbonyl diimidazole. In this work they were utilized to shape consideration edifices with three distinct gases for example 1-methylcyclopropene, oxygen and carbon dioxide. The exemplification of gas was demonstrated by direct response to known adduct (1methylcyclopropene), by gravimetric investigation (CO2) and by oxy meter (Oxygen).
The complexation of oxygen or carbon dioxide could be valuable for some biomedical applications. Specifically, the oxygen-filled nano sponges could supply oxygen to the hypoxic tissues which are available in different illnesses. 1methylcyclopropene remembered for $\beta$-cyclodextrin Nano sponges showed predominant ant ethylenic exhibitions in enduring cut blossoms in correlation with advertised products. ${ }^{13}$

\subsection{Carbamate nano sponges}

Such NS arranged by dissolvable strategy under an anhydrous/nitrogen air at room temperature to $70^{\circ} \mathrm{C}$ is gotten by responding cyclodextrins with cross-linkers, for example, hexamethylene diisocyanate and toluene diisocyanate in a molar proportion of $1: 2$ to 1:8. Originally combined by DeQuan Li and Min] in 1998, such NSs were produced for the treatment of water, including the expulsion of disintegrated natural carbon, for example, nitrophenol. The last advance is to plan atomically engraved nano sponges for the embodiment of substances like steroids, colours and dextromethorphan utilizing diisocyanate crosslinkers. ${ }^{13}$

\subsection{Anhydride Nano sponge}

Anhydride Nano sponge can be set up by the dissolvable technique within the sight of a base like pyridine or triethylamine to speed up the polymerization by utilizing crosslinkers, for example, pyromellitic dianhydride ethylenediamine tetra-acidic corrosive dianhydride of the cyclodextrins at room temperature Cyclodextrin: crossconnecting molar proportions going from 1: 2 to 1: 8 are utilized for arrangement. Various examinations have been led to epitomize different medications like doxorubicin, meloxicam, ibuprofen and acetylsalicylic corrosive.

\subsection{Epichlorohydrin cyclodextrin nano sponges}

These NSs arranged by dissolving cyclodextrins in an essential medium, for example, sodium hydroxide utilizing cross-connecting specialists, for example, epichlorohydrin are more hydrophilic in nature. Such nano sponges which display high compound opposition and flexible expanding capacity have been utilized to exemplify medications, for example, creatinine and captopril, enalapril, cilazapril, however in certain examinations a cross-connecting molar proportion of up to $1: 10$ with cyclodextrin has been considered. 13

9.5. CD-based ester nano sponges: A correct dianhydride as anhydride of pyromellitic utilised as crosslinking agent for production of such kind of Nano sponges. The heat release of crosslinking riposte is very fast [over in 5 min] and undergoes at normal temperature, $C D$ is dissolved in presence of pyridine. This kind of nano sponges have both anionic and cationic nature of free carboxylic acidic group.

9.6. Polyamide Nano sponges: Such variant of Nano sponges manufactured by carrying riposte in acrylamide at $94 \mathrm{hrs}$ in normal temperature. These bulge in aqueous solution and in both basic and acid remained solutions. It forms a transparent gel in contact with solution of water. Time contingent bulging on standard stability of gel for $80 \mathrm{hr}$. These preparations were undergone by utilising the albumin as protein with high amount of entrapment, around 95\%. invitro studies shows protein like albumin release at harmonize at $24 \mathrm{hr}$. Sodium lauryl sulphate [SDS] page formulation utilized to find stability of solution. This shows produced formulation stable for several months. 


\section{Characterization of nano sponges:}

The portrayal techniques for the complexed drug/nano sponges are recorded beneath:

10.1. Dissolvability considers: Consideration buildings is a method by which can decide the dissolvability and bioavailability of the medication. This strategy is the most broadly moved toward method for examination of the incorporation complex of nano sponges. Level of fruition can be known by the plot of stage solvency. Dissolvability contemplates are led to get to the $\mathrm{pH}$ of the medication, solubilization diagram and to assess the components influencing drug solvency.

10.2. Microscopic studies: Scanning Electron Microscopy (SEM) and Transmission Electron Microscopy (TEM) can be used to study the microscopic aspects of the drug, nano sponges and the product (drug/nano sponge complex). The difference in crystallization state of the raw materials and the product seen under electron microscope indicates the formation of the inclusion complexes 14 .

10.3. Zeta potential: Zeta potential can be characterized as the distinction of potential between two layers (scattering medium and fixed layer) of liquid secured with scattered particles. Zeta potential is the significant key marker for the strength of the colloidal scattering. By including additional anode molecule size hardware or zeta seizer, the zeta potential can be estimated. Higher the estimation of zeta capability of a colloidal scattering more is its solidness.

10.4. Thermal analytical methods: In the event that any progressions happen in medication atoms or particles goes through certain progressions prior then the warm debasement of nano sponges it very well may be dictated by the thermo-compound technique. The progressions of medication particles can be softening, dissipation, oxidation and disintegration and polymeric changes. The adjustments in the medication atoms demonstrate the arrangement of a decent intricate.

10.5. Molecule size and polydispersity: Particle size is dictated by the cycle of dynamic light dispersing utilizing 90Plus molecule size deciding programming. Dynamic light dispersing (DLS) is characterized as a procedure used to discover the size dissemination profile of nanoparticles. Finally, the last measurement of the particles and polydispersity record (PDI) can be found.

10.6. Thin layer chromatography (TLC): Attention can be characterized as a method which can be utilized to isolate the non un predictable or evaporative combination. In this strategy, if the Rf estimation of a specific medication atom is of a satisfactory reach then it is useful in perceiving the arrangement of a complex among drug and nano sponges 15 .

10.7. Infrared spectroscopy: The collaboration between Nano sponges and the medication in the strong state can be controlled by utilizing infrared spectroscopy. Nano sponge particles appended in the edifices which are under $25 \%$, the medication range can be effectively veiled by the range of nano sponges. The strategy isn't fitting to distinguish the incorporation complex over different strategies 15

\section{Pharmaceutical use of Nano sponges:}

Due to their biocompatibility and adaptability, nano sponges have numerous applications relating the drug field. Nano sponges can be utilized as excipients in planning of tablets, cases, pellets, granules, suspension, strong scattering or effective dose structures.
Topical treatment is an appealing decision for the treatment of the coetaneous diseases because of different benefits, for example, focusing of medications to the immediate site of contamination and decrease of fundamental results. Econazole nitrate (imidazole) is an antifungal or drug fungicide utilized topically to fix competitor's foot, ringworm, tinea pityriasis versicolor, athlete tingle and vaginal thrush. The accessible results of econazole nitrate present in the market are cream, balm, salve, and arrangement. Adsorption of econazole nitrate isn't critical when it is applied to the skin and viable treatment; need a high convergence of dynamic specialists to be joined. Consequently, econazole nitrate nano sponges were manufactured by emulsion dissolvable technique and these econazole nitrate nano sponges were stacked in a hydrogel as a skin conveyance for supported arrival of the medication 16 .

Itraconazole is additionally an antifungal medication goes under biopharmaceutical order framework class II and that has a disintegration rate restricted and helpless bioavailability. So, the point of this investigation was to expand the solvency of the itraconazole, so that can tackle the bioavailability issue. In these nano sponges, whenever utilized $\beta$-cyclodextrin as cross-connected with carbonate bonds and stacked it with itraconazole than the dissolvability of itraconazole can be increased. ${ }^{17}$

Nano sponges, arranged utilizing two distinctive crosslinkers on Curcumin (CUR) which has a poor watery dissolvability of photoreactive and intense anticancer action. For this reason, diverse molar proportions of beta cyclodextrin with diphenyl carbonate (DFC) and pyromellitic dianhydride (PMDA) were applied by dissolvable technique to get 1:2 ideal molar proportion of two distinctive NS and afterward CUR was coordinated into NSs with 1:1 molar proportion. Medication discharge, security, solvency and cytotoxicity estimations of NS were contrasted and unadulterated medication and concerning one another. Appropriately, the dissolvability of PMDA crosslinked drug stacked NS (PMDA-CUR-NS) was $63.98 \%$, while the solvency of DFC crosslinked drug stacked NS (DFC-CUR-NS) was $18.6 \%$. in vitro drug arrival of PMDA-CUR-NS expanded 16 crease contrasted with unadulterated CUR and 5-overlap contrasted with DFC-CUR-NS, while the in vitro cytotoxicity IC50 estimation of PMDA-CUR-NS diminished 2.2 occasions contrasted with unadulterated medication and diminished 1.5 occasions looked at DFC-CUR-NS. Accordingly, they stressed that PMDA-NS is a more potential nanocarrier contrasted with DFCNS.18

\section{Conclusion:}

Nano sponges are drug conveyance framework which can convey both hydrophilic and hydrophobic medications. They can be figured in various detailing, for example, oral, parenteral and effective framework. Nano sponge innovation has wide application in Pharmaceutical industry. Medications created by this innovation give delayed and controlled arrival of the medication in a protected and powerful manner.

\section{References}

[1] Bhatia S. Nano sponges Types, Classification, Characterization, Fabrication Methods and Drug Delivery Applications. Natural Polymer Drug Delivery Systems, Springer International Publishing Switzerland, 2016.

[2] Thakre AR, Gholse YN, Kasliwal RH. Nano sponges: a novel approach of drug delivery system. J Med Pharm Allied Sci 2016; 78:103-111.

[3] Dhavala PB, Kumar VS. An Interesting Nano sponges as a Nanocarrier for Novel drug delivery: A Review, Int J of Pham and Med Res. 2017; 5(2). 
[4] Khan KA, Bhargav E, reddy KR, Sowmya C. Nano sponges: A New Approach for Drug Targeting. Int. J pharm. pharm. res. 2016; $7(3): 381-396$.

[5] Majeed A, Khan NA, Ocular in situ gel: An overview. Journal of Drug Delivery and Therapeutics, 2019; 9(1):337-347. https://doi.org/10.22270/jddt.v9i1.2231.

[6] Suchita G. Waghmare, Rasika R. Nikhade, Dr. Satish and B. Kosalge. Nanosponges:a novel approach for controlled release drug delivery system.InternationalJournal of Pharmacy and Pharmaceutical research, 2017; 9(3):101-11.

[7] Shende, P.; Chaphalkar, R.; Deshmukh, K.; Gaud, R.S. Physicochemical Investigation of Engineered Nanosuspensions Containing Model Drug, Lansoprazole. J. Dispers. Sci. Technol. 2016; (37):504-511.

[8] Jyoti P, Tulsi B, Popin K, Chetna B. An Innovative Advancement for Targeted Drug Delivery: Nanosponges, Indo Global J Pharm Sci. 2016; 6(2):59-64.

[9] Arshad K, Khan A, Bhargav E, Reddy K and Sowmya C: Nanosponges: a new approach for drug targeting. International Journal of Advances in Pharmaceutical Research 2016; 7(3):38196.

[10] Himanghshu Bhowmik, D. Nagasamy venkatesh, Anuttam Kuila Kammari Harish kumar international journal of pharmaceutical sciences 2018:10(4).

[11] Sabzi NE and Kiasat AR: $\beta$-Cyclodextrin based nanosponge as a biodegradable porous three-dimensional nanocatalyst in the one-pot synthesis of n-containing organic scaffolds. Catalysis Letters 2018: 1-11.

[12] Osmani RA, Kulkarni P, Manjunatha S, Gowda V, Hani U, Vaghela $\mathrm{R}$ and Bhosale R: Cyclodextrin Nanosponges in Drug Delivery and Nanotherapeutics. Environmental Nanotechnology 2018: 279-42.

[13] Bergal A, Elmas A, Akyüz G .A New Type and Effective Approach for Anti-Cancer Drug Delivery Application - A Nanosponge. Nano Res Appl ,I Med Pub journal; 2019; 3(1).

[14] Jyoti P, Tulsi B, Popin K, Chetna B. An Innovative Advancement for Targeted Drug Delivery: Nanosponges, Indo Global J Pharm Sci. 2016; 6(2):59-64.

[15] System Pawar AY, Naik AK, Jadhav KR, Nanosponges: A Novel Drug Delivery, Asian Journal of Pharmaceutics .2016; 10 (4).

[16]. Darandale SS, Shevalkar GB and Vavia PR: Effect of lipid composition in propofol formulations: decisive component in reducing the free propofol content and improving pharmacodynamic profiles. AAPS Pharm Sci Tech 2017; 18(2):441-50.

[17]. Kadian R. Nanoparticles: a promising drug delivery approach. Asian J Pharm Clin Res 2018; 11:30-5.

[18]. Pushpalatha R, Selvamuthukumar S, Kilimozhi D. Cross-linked, cyclodextrin-based nanosponges for curcumin deliveryphysicochemical characterization, drug release, stability and cytotoxicity. J Drug Deliv Sci Technol 2018; 45-53. 